\title{
Single-nucleotide polymorphisms of hNEIL2 gene: from protein structure to functions in base excision DNA repair
}

\author{
Zarina Kakhkharova \\ ICBFM SB RAS, Novosibirsk, Russia \\ Novosibirsk State University, \\ Novosibirsk, Russia \\ zarinkaapels@gmail.com
}

\author{
Petrova Daria \\ ICBFM SB RAS, Novosibirsk, Russia \\ Novosibirsk State University, \\ Novosibirsk, Russia \\ dashapetr21@gmail.com
}

Inga Grin

ICBFM SB RAS, Novosibirsk, Russia

Novosibirsk State University,

Novosibirsk, Russia

grin@niboch.nsu.ru

\begin{abstract}
Base excision repair (BER) is one of most universal mechanisms of DNA repair in living cells. BER initiated by specific enzymes - DNA-glycosylases recognizing damaged nucleotide and can influence on efficiency of all DNA repair pathway. Nucleotide polymorphisms (SNPs) of the NEIL2 gene could be associated with the risk of cancer develope in actively transcribed organs. We found two SNPs located in conservative regions responsible for catalysis and DNA binding and purified corresponding recombinant protein variants hNEIL2(R103W) and hNEIL2(P304T), respectively. A comparison of the enzymatic characteristics with the wild-type hNEIL2 protein revealed reducing enzyme activities for hNEIL2(P304T).
\end{abstract}

Keywords - NEIL2, base excision repair, DNA-glycosylase, single nucleotide polymorphism

\section{Motivation and Aim}

Every day in our cells the DNA molecule is chemically modified by numerous exogenous and endogenous factors. To correct these damages there are same protective mechanisms named DNA repair and base excision repair (BER) is one of most universal. BER is initiated by specific enzymes DNAglycosylases capable to recognize and excise the modified DNA bases with followed by some enzymatic stages for recovering intact structure of DNA. Gene sequence variation like a single-nucleotide polymorphism (SNP) can lead to another polypeptide sequence and enzymatic activity changes. Therefore, SNPs in DNA-glycosylase genes can decrease efficiency of BER and promote DNA damages accumulation causing the oncological transformation of cells.

Mammalian DNA-glycosylase NEIL2 is a bifunctional enzyme with AP-lyase and glycosylase activity [1]. Additionally NEIL2 has unique ability to recognize oxidative damages in "bubble" structures [2] forming during transcription. On the other hand, it is known that some SNPs of hNEIL2 gene are associated with risk of cancer developing in actively transcribed organs particular, one of the most dangerous - lung cancer. This project aims to study the effect of SNPs in the conserved regions of the hNEIL2 gene on the enzymatic functions of protein variants. This understanding can provide a real opportunity to predict an increased cancer risk in carriers of the corresponding alleles.

\section{Methods}

We analyzed the available SNP databases hNEIL2 gene and the structure of the hNEIL2 protein using bioinformatics methods (since XRC data are not available) and found that some SNP lead to large changes in the protein structure. We cloned the wild type gene and SNP corresponding genes using standard mutagenesis methods, expressed and purified recombinant proteins by affinity chromatography. Then, the substrate specificity and enzyme activities were studied for hNEIL2 recombinant proteins.

\section{Results}

We found that two SNPs are located in the coding region of the gene and alter the class of amino acids in conservative regions responsible for catalysis and DNA binding, hNEIL2(R103W) and hNEIL2(P304T) respectively. According to the 1000 genome and ExAC projects the frequencies of these alleles were equal to MAF (Minor Allele Frequency $)=0.0148$ for hNEIL2 (R103W) and MAF $=0.0088$ for hNEIL2(P304T). All proteins exhibit increased DNA glycosylase activity observed in the following chain: doublestranded DNA, single-stranded DNA and DNA with "bubble" structure. The hNEIL2(P304T) polymorphic variant dramatically reduces both AP-lyase and DNA glycosylase activities.

\section{ACKNOWLEDGMENT}

This study has been supported by RFBR according to the research project No. 18-44-540029.

\section{REFERENCES}

[1] Grin I., Zharkov D.O. (2010) Inactivation of NEIL2 DNA glycosylase by pyridoxal phosphate reveals a loop important for substrate binding. Biochemical and Biophysical Research Communications Res. 394(1): 100-105.

[2] Makasheva K.A., Zharkov D.O. Requirements for DNA bubble structure for efficient cleavage by helix-two-turn-helix DNA glycosylases. Mutagenesis 35(1): 119-128. 